\title{
Spectral meta-moments reveal hidden signatures of vortex pulses
}

\author{
Max Liebmann ${ }^{1}$, Alexander Treffer ${ }^{1}$, Martin Bock ${ }^{1}$, Thomas Seiler ${ }^{2}$, Jürgen Jahns ${ }^{2}$, \\ Thomas Elsaesser ${ }^{1}$, and Ruediger Grunwald ${ }^{1}$ \\ ${ }^{1}$ Max Born Institute for Nonlinear Optics and Short-Pulse Spectroscopy, Max Born Str. 2a, \\ 12489 Berlin, Germany \\ ${ }^{2}$ FernUniversität Hagen, Micro- and Nanophotonics, Universitätsstr. 27/PRG, 58097 Hagen, Germany
}

\begin{abstract}
Ultrashort vortex pulses possess specific spatio-spectral signatures due to Gouy phase shift. Helical movement of anomalies around the phase singularity was found which is referred to as spectral Gouy rotation. The analysis of spectral moments allows for identifying related characteristic patterns even in weakly modulated spectral maps. Radial meta-moments from polar projections deliver information on global spectral gradients and oscillatory behavior. The particular mathematical tools could also be applied to other fields of ultrafast spectroscopy.
\end{abstract}

\section{Introduction}

Ultrashort pulses with orbital angular momentum (OAM) are highly relevant for new applications in physics, chemistry and information technology by increasing the usable degrees of freedom. Polychromatic light fields with OAM like ultrashort vortex pulses show characteristic spectral anomalies in the vicinity of phase singularities $[1,2]$. Their spectral properties are still the subject of fundamental research and challenging with respect to the techniques of experiment and data processing. Here we report on spatial-spectral mapping of femtosecond vortex pulses in combination with advanced analysis techniques based on spectral meta-moments. The capability of this approach is demonstrated by extracting peculiar propagation features of vortex pulses in spectral domain which remain completely hidden for unspecific detection techniques like intensity monitoring.

\section{Experimental and mathematical techniques}

Experiments were focused to the study of the rotational and oscillatory behavior of two-dimensional spatio-spectral maps of propagating femtosecond Bessel-Gauss-type vortex pulses with so-called "spectral eyes", i.e., extremal points (peaks and holes) of spectral landscapes. This phenomenon results from the complex interplay of spectraldependent Gouy phase shift, spectral profile and wavefront twist and are referred to as "spectral Gouy rotation" [3]. The experimental study is particularly difficult because the relevant effects appear within dark zones near singularities where spectral 
modulations are weak. Therefore, the identification of characteristic spatial signatures requires sensitive spectroscopic methods with high selectivity.

\subsection{Experimental setup}

In the experiments, spectral maps of 120 -fs Ti:sapphire vortex pulses are detected at different propagation distances with a scanning fiber-coupled spectrometer moved by an $x$-y-translation stage. By representing local spectra by statistical moments, we extract essential features like spectral Gouy rotation of spectral anomalies from propagation sequences in this first approach. The moment maps, however, indicate a far richer structure of spatio-spectral chirp which calls for a more detailed analysis. For this, a statistical analysis method based on particular functionals is of interest, the so-called radial meta-moments (RMM) which are (in contrast to the local moments) sensitive for global spectral gradients of 2D maps [4].

\subsection{Spectral moments and meta-moments}

Local spectral moments select particular shape parameters depending on the order $j$ of the moment ( $j=1-4$ : mean, variance, skewness, kurtosis). Propagation sequences of 2D moment maps, however, contain information about the complex spatial spectral transport of intensity in wavepackets with OAM including shape deformation (symmetry parameters), size variation (expansion, shrinking) and rotation (swirl). The spatial variation of the moments can be described quantitatively by metamoments in spectral domain that are defined as the statistical moments of spectral statistical moments. For radial cuts through maps of $j^{\text {th }}$ moments at a propagation distance $z$, centred at a phase singularity and plotted in polar coordinates (angle $\varphi$, radial distance $r$ ), radial meta-moments (RMMs) of the order $k$ can be calculated [4]:

$$
\mu_{r}^{(k, j)}(\varphi, z)=M_{r}^{(k)}\left[M^{(j)}(r, \varphi, z)\right]
$$

\section{Results and discussion}

The analysis of RMMs for different distances $z$ reveals vortex-specific spectral propagation dynamics. To illustrate this, the case of $k=2$ and $j=1$ was studied for two "spectral eyes" (minimum and maximum of first moments) of a vortex pulse (120-fs Ti:sapphire laser, $10 \mathrm{~nm}$ spectral FWHM). Spectral center-of-gravity maps for two different distances are plotted in Figs. 1a,b. One can recognize the changing extension of the extremal areas. The representation by $\mu_{r}(2,1)(z)$ for 4 different angles $\left(\varphi=270^{\circ}\right.$ $300^{\circ}$ ) is shown in Fig. 1c. The variance of the local spectral centers of gravity was found to vary with increasing propagation distance. The speed of change is not constant and reaches maximum values on the time scale of few-ten femtoseconds around distinct flipping points. The size of the "spectral eyes" (here: the spectral minimum) increases rapidly (shortest interval for maximum enlargement: $34 \mathrm{fs}$ ).

To conclude, spatio-spectral properties of femtosecond vortex pulses close to the phase singularity were characterized with advanced statistical methods. The capability of radial meta-moments (RMMs) for filtering out specific spectral information in ultrafast singular optics with enhanced selectivity was demonstrated. RMM analysis allows for identifying significant modes of propagation and distortions of vortex pulses which are imprinted in the geometry of extremely weak spatial 
gradients in 2D spectral maps. Currently, the prospects of adaptive moment analysis are investigated. By introducing variable radii in the meta-moment procedures, the spatial horizon for the characterization of spectral or temporal maps can be matched to their particular fine structure, e.g. in the presence of complex arrangements of anomalies. Moreover, the approach of meta-moment promises to find further applications in spectroscopy. For example, contrast enhancement and statistical selectivity could improve the extraction of essential features in two-dimensional molecular spectroscopy and spectral microscopy.
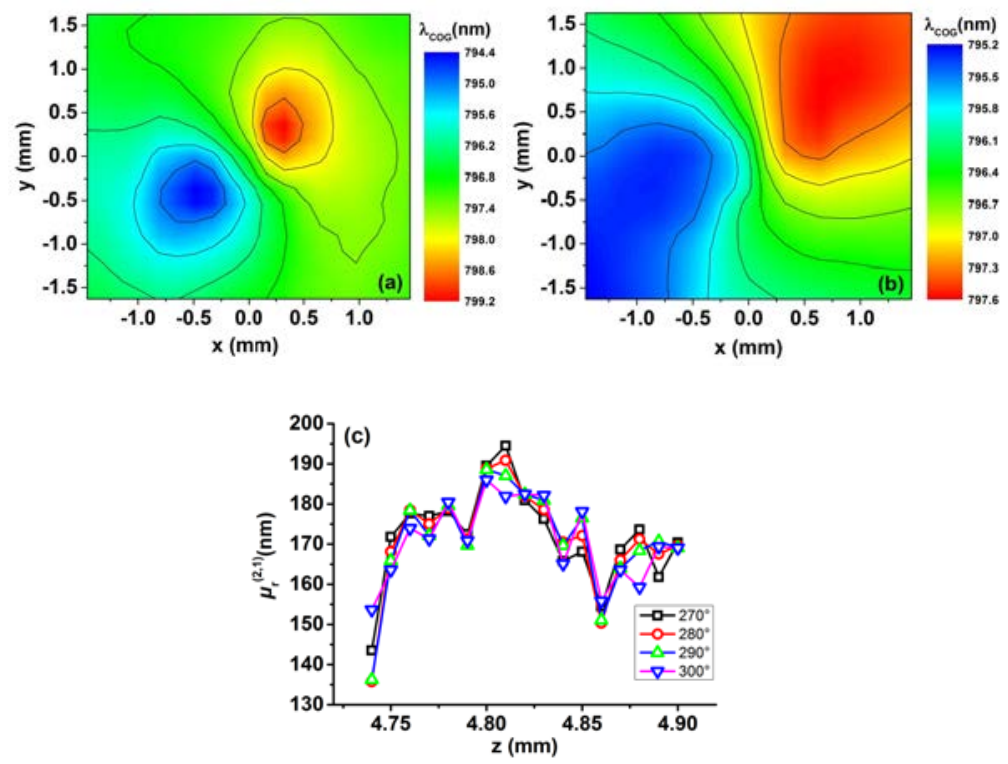

Fig. 1. Fast spatial switching of the size of spectral eyes of a propagating vortex pulse generated by a dispersion compensated diffractive spiral phase plate; $(a, b)$ : 2D spectral center-of-gravity maps for two "spectral eyes" detected at $z=4.78$ and $z=4.82 \mathrm{~mm}$, respectively. To visualize size-related features of spectral propagation characteristics, second-order radial meta-moments (RMMs) at 4 cut angles $\left(270^{\circ}-300^{\circ}\right)$ were calculated for maps of first order spectral moments detected at varying axial distance $z$. The time interval for maximum increase of the RMM value (size parameter) was found to be less than $34 \mathrm{fs}$.

The authors gratefully acknowledge theoretical simulations by M. Musigmann (FernUniversität, Hagen), assistance for using field propagation software by LightTrans (Jena), mask fabrication by Photronics (Dresden), and close collaboration by U. Wallrabe (IMTEK, University Freiburg). The work was financially supported by DFG (GR 1782/14-2).

\section{References}

1. M. V. Berry, New J. Phys. 4, 66.1-66.14 (2002)

2. J. Leach, M. J. Padgett, New J. Phys. 5, 154.1 (2003)

3. M. Liebmann, A. Treffer, M. Bock, T. Elsaesser, R. Grunwald, Opt. Express 25, 26076-26088 (2017)

4. M. Liebmann, A. Treffer, M. Bock, T. Seiler, T. Elsaesser, R. Grunwald, in: Eds E. J. Galvez, D. L. Andrews, J. Glückstad, Proc. SPIE 10549, 10549-14 (2018) 